\title{
Effect of surface tension on the behavior of adhesive contact based on Lennard-Jones potential law
}

\author{
Xinyao Zhu, Wei Xu* \\ Faculty of Engineering and Physical Sciences, University of Surrey, Guilford GU2 7XH, UK \\ Corresponding author: Tel: +44 (0)148368 2368,E-mail:w.xu@surrey.ac.uk
}

\begin{abstract}
The present study explores the effect of surface tension on adhesive contact behavior where the adhesion is interpreted by long-range intermolecular forces. The adhesive contact is analyzed using the equivalent system of a rigid sphere and an elastic half space covered by a membrane with surface tension. The longrange intermolecular forces are modelled with the Lennard-Jones $(\mathrm{L}-\mathrm{J})$ potential law. The current adhesive contact issue can be represented by a nonlinear integral equation, which can be solved by Newton-Raphson method. In contrast to previous studies which consider intermolecular forces as short-range, the present study reveals more details of the features of adhesive contact with surface tension, in terms of jump instabilities, pull-off forces, pressure distribution within the contact area, etc. The transition of the pull-off force is not only consistent with previous studies, but also presents some new interesting characteristics in the current situation.
\end{abstract}

Keywords: Adhesive contact; surface tension; Lennard-Jones (L-J) potential; instability; Hysteresis; Pulloff force

\section{Introduction}

Surface adhesion is of significant importance not only in natural objects but also in many engineering systems, such as microelectromechanical, nanoelectromechanical devices and atomic force microscopy (AFM) where surface forces dominate bulk forces. In surface adhesion, the fundamental problem is the adhesive contact between two bodies. One of the precursive investigations on adhesive contact is ascribed to Bradley (1932), who adopted the Lennard-Jones potential to describe the adhesive forces between two rigid spheres. The studies on adhesive contact between two elastic spheres were initially performed by Johnson et al. (1971) who formulated the relation between applied load and the contact radius, known as JKR theory, and later the contact edge in the adhesive contact problem was treated as the cusp of an external crack.

Although the JKR theory has possessed success in predicting the adhesive contact behavior of elastic spheres, it is lately found that the adhesion-induced deformation of soft substrates, e.g. plasticized polystyrene (Rimai et al., 2000), hydrogels (Chakrabarti et al., 2013) and silicone gels (Style et al., 2013) diverges significantly from that described by JKR theory. For example, Style et al. (2013) found that the relation between contact radius and sphere radius is analogous to that predicted by the Young-Dupre law rather than JKR theory, as either the sphere radius or the elastic modulus of substrate reduces. Style et al. (2013) ascribed this divergence to the exclusion of the substrate-air surface tension which also resists the adhesion-induced deformation by flattening the surface of soft solids.

Owing to this deviation, some recent studies began to investigate the effect of surface tension on the adhesive contact. Salez et al. (2013) probed the adhesive contact between an elastic sphere and a rigid plane under zero external load with surface tension, and they realized a continuous bridge between the JKR and Young-Dupre asymptotic regimes. Using the finite element method including the surface tension and 
hyperelasticity, Xu et al. (2014) simulated the adhesive contact between a rigid sphere and a neo-Hookean substrate in the absence of external load, and showed the transition between the classical Johnson-KendallRoberts (JKR) deformation and a liquid-like deformation. Cao et al. (2014) verified this transition via simulations realized by molecular dynamics. Hui et al. (2015) studied the impact of surface tension on the non-slip adhesive contact between a rigid sphere and an incompressible substrate, and proposed a single dimensionless parameter: $\omega=\sigma(G R)^{-2 / 3}(9 \pi \Delta \gamma / 4)^{-1 / 3}(\sigma, G, R$ and $\Delta \gamma$ denote surface tension, shear modulus of the substrate, sphere radius and the interfacial work of adhesion respectively) to characterize the transition between JKR theory and Young-Dupre law. Long et al. (2016) reconsidered the same problem studied by Hui et al. (2015), but without the requirement of non-slip and incompressibility of substrate material, and they presented an explicit relation between the contact radius and the indent depth in the absence of external load, which are more convenient in practical applications.

In all the experimental and numerical studies mentioned above, the surface adhesion is interpreted by short-range intermolecular forces (in fact, intermolecular forces are consider as a delta function in JKR theory), and thus the adhesive forces vanish outside the contact area. Since the short-range intermolecular forces are merely an approximation of their actual counterpart which are generally long-range, it is of great necessity to reconsider the effect of surface tension on the adhesive contact which is subject to more realistic molecular interactions, e.g. van der Waals forces. On the other hand, the success of Lennrad-Jones (L-J) potential in describing the adhesive contact behavior of elastic bodies without surface tension by Greenwood (1997) and Feng (2000) also provides us with sufficient fundamental technique to apply the L-J potential law to the current issue.

In the present study, we aim to explore the effect of surface tension on the behavior of adhesive contact where the adhesive interaction obeys the Lennard-Jones potential law. In the next section, we firstly reintroduce the Green's function of a point force acting on an elastic substrate with surface tension, and show that the current adhesive contact problem can be transformed into the solution of an integral equation. Section 3 presents computational techniques to solve this integral equation. Discussions are presented in Section 4. Concluding remarks are provided in Section 5.

\section{Adhesive contact with surface tension}

In the surface elasticity theory (Gurtin and Murdoch, 1975; Gurtin et al., 1998), the surface is treated as an inappreciably thin membrane ideally adhered to the bulk material. Analogous to classical theory of solid mechanics, the elastic surface also has its equilibrium and constitutive equations. For a flat surface, the equilibrium conditions are given by (Chen et al., 2006; Mogilevskaya et al., 2011):

$$
\begin{gathered}
t_{\alpha}+\sum_{\beta=1}^{2} \sigma_{\beta \alpha, \beta}^{s}=0(1) \\
\sum_{i=1}^{3} \sum_{j=1}^{3} \sigma_{i j} n_{i} n_{j}=\sum_{\alpha=1}^{2} \sum_{\beta=1}^{2} \sigma_{\alpha \beta}^{s} \kappa_{\alpha \beta}(2)
\end{gathered}
$$

where $t_{\alpha}(\alpha=1,2)$ is the surface traction in $x_{\alpha}$ direction, $n_{i}$ is the unit vector normal to the deformed surface, $\kappa_{\alpha \beta}$ is the curvature tensor of the surface and $\sigma_{\alpha \beta}{ }^{s}$ is the surface stress tensor. Cammarata (1994) presented the relationship between the surface stress tensor $\sigma_{\alpha \beta}{ }^{s}$ and surface energy density $\sigma\left(\varepsilon_{\alpha \beta}\right)$, which could be viewed as the constitutive equations for surface elasticity, as

$$
\sigma_{\alpha \beta}^{s}\left(\varepsilon_{\alpha \beta}\right)=\sigma\left(\varepsilon_{\alpha \beta}\right) \delta_{\alpha \beta}+\frac{\partial \sigma\left(\varepsilon_{\alpha \beta}\right)}{\partial \varepsilon_{\alpha \beta}}(3)
$$

where $\delta_{\sigma \beta}$ and $\varepsilon_{\alpha \beta}$ denote Kronecker delta function and surface strain tensor respectively. If the change of the atomic spacing in deformation is infinitesimal, the contribution of the second term in Eq. (3) to the surface stress tensor is negligibly small compared to the surface energy (Yang, 2004; Shenoy, 2005), and hence one has

$$
\sigma_{\alpha \beta}^{s}=\sigma\left(\varepsilon_{\alpha \beta}\right) \delta_{\alpha \beta}(4)
$$


Since atomic simulation indicates that the surface energy of nanoparticles almost remains constant when the radius of nanoparticles is larger than $4 \mathrm{~nm}$ (Bian et al., 2012), we assume that surface energy density $\sigma$ is constant on surface in the present study, resulting in a constant residual surface tension, which can capture the main surface effects in contact mechanics (Gao et al., 2013). It is worth noting that the surface elasticity theory by Gurtin and Murdoch (1975) (G-M model) remains as an open topic, and later it is followed by subsequent new studies by such as Huang and Wang (2006) who developed a hyperelastic surface model, and Chen and Yao (2014) who proposed the concept of surface energy density to characterize surface elasticity.

Based on the following hypotheses:

1. The surface tension $\sigma$ in the membrane mentioned above is large enough such that it is not changed appreciably after being subject to small deformations.

2. The surface is in frictionless contact with the bulk material.

Hajji (1978) developed an analytical solution for the normal stress $\sigma_{z}$ and displacement $(u, w)$ of a semiinfinite space subject to a vertical concentrated force $P$, with the foregoing surface tension:

$$
\begin{gathered}
\sigma_{z}(r, 0)=\frac{P}{2 \pi s}\left\{\frac{\pi}{2 s}\left[H_{0}\left(\frac{r}{s}\right)-Y_{0}\left(\frac{r}{s}\right)\right]-\frac{1}{r}\right\}(5) \\
u(r, 0)=-\frac{(1-2 v) P}{4 \pi G} \int_{0}^{\infty} \frac{J_{1}(r t)}{1+s t} d t(6) \\
w(r, 0)=\frac{P}{4 \sigma}\left[H_{0}\left(\frac{t}{s}\right)-Y_{0}\left(\frac{t}{s}\right)\right](7)
\end{gathered}
$$

where $s=\sigma(1-v) / G$, is an intrinsic material length indexing the relative significance of surface tension. $G$ and $v$ denote the shear modulus and Poisson's ratio of the half space respectively. $H_{n}(\cdot), J_{n}(\cdot)$ and $Y_{n}(\cdot)$ denote the Struve function, Bessel function of the first kind and Bessel function of the second kind, respectively, and $n$ is the order.

Now we begin to consider the adhesive contact of a rigid sphere with radius $R$ indenting on an elastic half space, as illustrated in Fig. 1. The half space is covered with the aforementioned membrane with surface tension, and the adhesive interaction between the sphere and half space obeys the Lennard-Jones (L-J) potential law.

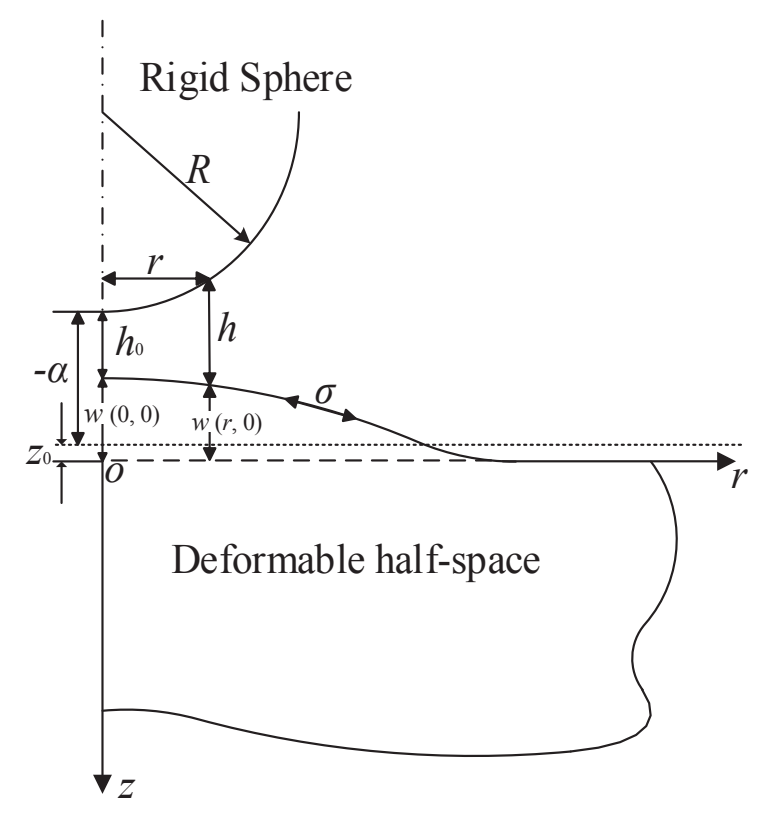


Fig. 1. Schematic of a rigid sphere in close proximity with an elastic half space with surface tension, where the pile-up at the upper half space is due to the adhesion effect.

According to the geometry illustrated in Fig. 1, the gap $h(r)$ between the sphere and the half space is:

$$
h(r)=-\alpha+z_{0}+\frac{r^{2}}{2 R}+w(r, 0)(8)
$$

where $z_{0}$ denotes the "equilibrium distance" between two parallel and flat surfaces at which the net local intermolecular force is zero; $\alpha$ is the approach of the sphere with respect to the zero force position $z=-z_{0}$; $w(r, 0)$ stands for the deflection of the surface, and positive and negative $w$ represents downward and upward deflection respectively. Ascribed to the surface interaction controlled by the $\mathrm{L}-\mathrm{J}$ potential, the local pressure $p(r)$ is described by (Israelachvili, 1992):

$$
p(r)=-\frac{8 \Delta y}{3 z_{0}}\left\{\left[\frac{z_{0}}{h(r)}\right]^{3}-\left[\frac{z_{0}}{h(r)}\right]^{9}\right\}(9)
$$

where $\Delta y$ denotes the magnitude of work needed to separate two surfaces to infinity.

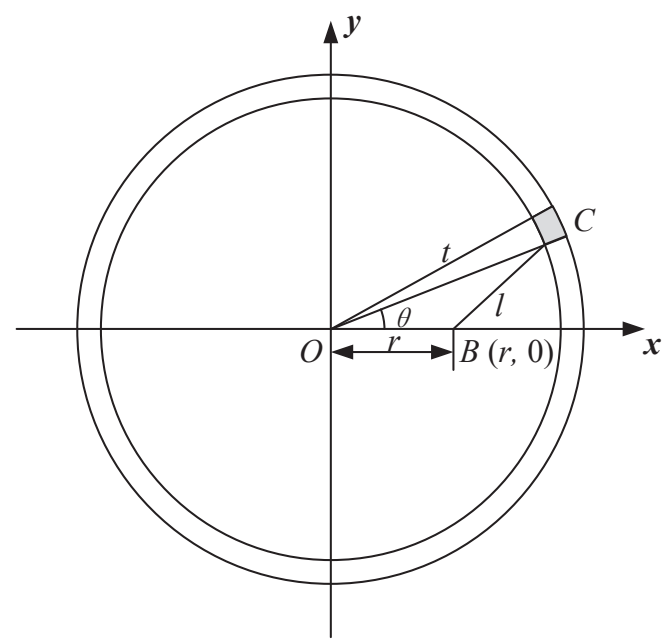

Fig. 2. Schematic of the planform of the adhesive contact.

Fig. 2 illustrates the plan view of the adhesive contact. The normal displacement $d w(r, 0)$ at an arbitrary point $B(r, 0)$ induced by the pressure $p(t)$ acting on an infinitesimal surface element of area $t d \theta d t$ at another arbitrary point $C(t, \theta)$ is given by:

$$
d w(r, 0)=\frac{p(t) t d \theta d t}{4 \sigma} \psi\left(\frac{l}{s}\right)(10)
$$

with $l=\left(r^{2}+t^{2}-2 r t \cos \theta\right)^{1 / 2}, \psi(x)=H_{0}(x)-Y_{0}(x)$. Thus the total normal displacement at point $B$ due to the pressure acting on the whole surface should be:

$$
w(r, 0)=\int_{\theta=0}^{\pi} \int_{t=0}^{\infty} \frac{p(t) t d \theta d t}{2 \sigma} \psi\left(\frac{l}{s}\right) d \theta d t(11)
$$

Combining Eq. (8), (9) and (11), we could obtain a single nonlinear integral equation for a single unknown $h(r)$, which describes the adhesive contact behavior with surface tension, as 


$$
h(r)=-\alpha+z_{0}+\frac{r^{2}}{2 R}-\frac{4 \Delta \gamma}{3 \sigma z_{0}} \int_{0}^{\infty} G\left(\frac{r t}{s^{\prime}-}\right) t\left\{\left[\frac{z_{0}}{h(t)}\right]^{3}-\left[\frac{z_{0}}{h(t)}\right]^{9}\right\} d t(12)
$$

where

$$
G\left(\begin{array}{r}
r t \\
s^{\prime} s
\end{array}\right)=\int_{\theta=0}^{\pi} \psi\left(\frac{\sqrt{r^{2}+t^{2}-2 r t \cos \theta}}{s}\right) d \theta(13)
$$

If one introduces the following dimensionless parameters: $H=h / z_{0}-1, A=\alpha / z_{0}, \rho=r / s, \tau=t / s$ and $\zeta=$ $\left(R z_{0}\right)^{1 / 2} / s$, Eq. (12) can be rewritten as

$$
\zeta H(\rho)+\zeta A-\frac{\rho^{2}}{2 \zeta}+\frac{8}{3} \mu^{3 / 2} \int_{\tau=0}^{\infty} G(\rho, \tau) \tau\left\{\frac{1}{[1+H(\tau)]^{3}}-\frac{1}{[1+H(\tau)]^{9}}\right\} d \tau=0(14)
$$

where $\mu=\left[R \Delta \gamma^{2} /\left(E^{* 2} z_{0}^{3}\right)\right]^{1 / 3}$ denotes the so-called Tabor parameter. In Eq. (14), if $\zeta$ and $\mu$ are fixed, the unknown $H(\rho)$ is solely dependent on the value of $A$. However, in the non-surface tension situation, both Greenwood (1997) and Feng (2000) pointed out that a single value of $\alpha$ might result in multiple values of $P$, and vice versa. This problem could be circumvented by parameterizing $P$ and $\alpha$ in terms of the central gap $H_{0}=H(0)$ (Greenwood 1997), which inspires us to adopt the same method in the present case with surface tension. By letting $\rho=0$ in Eq. (14), one will get

$$
\zeta H(0)+\zeta A+\frac{8}{3} \mu^{3 / 2} \int_{\tau=0}^{\infty} G(0, \tau) \tau\left\{\frac{1}{[1+H(\tau)]^{3}}-\frac{1}{[1+H(\tau)]^{9}}\right\} d \tau=0(15)
$$

from which $\zeta A$ could be replaced by the remaining terms. Substituting this replacement into Eq. (14) leads to

$$
\zeta H(\rho)-\zeta H(0)-\frac{\rho^{2}}{2 \zeta}+\frac{8}{3} \mu^{3 / 2} \int_{\tau=0}^{\infty} \operatorname{kern}(\rho, \tau) \tau\left\{\frac{1}{[1+H(\tau)]^{3}}-\frac{1}{[1+H(\tau)]^{9}}\right\} d \tau=0(16)
$$

where $\operatorname{kern}(\rho, \tau)=G(\rho, \tau)-G(0, \tau)$. The total applied force $P$ is calculated by integrating the pressure given in Eq. (9) with respect to the whole surface. If $P$ is scaled by $\pi R \Delta \gamma$, one gets:

$$
\frac{P}{\pi R \Delta \gamma}=-\frac{16}{3 \zeta^{2}} \int_{\tau=0}^{\infty} \tau\left\{\frac{1}{[1+H(\tau)]^{3}}-\frac{1}{[1+H(\tau)]^{9}}\right\} d \tau(17)
$$

By isolating the second term of Eq. (15), we could obtain the expression for the dimensionless approach $A$ :

$$
A=-H(0)-\frac{8 \mu^{3 / 2}}{3 \zeta} \int_{\tau=0}^{\infty} G(0, \tau) \tau\left\{\frac{1}{[1+H(\tau)]^{3}}-\frac{1}{[1+H(\tau)]^{9}}\right\} d \tau(18)
$$

From Eq. (17) and (18), it can be seen that the values of applied load $P$ and approach $\alpha$ can be derived once the dimensionless separation $H(\tau)$ is obtained.

\section{Computational details}

Although in theory, the integrating range of Eq. $(12)$ is $[0,+\infty)$, it is found to be justified to set the upper limit at $10\left(R z_{0}\right)^{1 / 2}$ (Feng, 2000), i.e. the upper limit of the integral in Eq. (14) is $10 \zeta$. In order to solve the nonlinear integral Eq. (14), the integrating range $[0,10 \zeta)$ is discretized by a finite number $(N+1)$ of nodes whose one-dimensional radial coordinate is $\rho_{i}(i=1,2, \ldots N+1)$, which generates a number of $N$ segments called elements. In this regard, the $N+1$ unknowns of $H_{i}=H\left(\rho_{i}\right)$ can be determined by solving $N+1$ independent residual equations $R_{i}=0$, where

$$
R_{i} \equiv \zeta H_{i}-\zeta H_{0}-\frac{\rho_{i}^{2}}{2 \zeta}+\frac{8}{3} \mu^{3 / 2} \sum_{j=1}^{N+1} \Delta \tau_{j} \tau_{j}\left[\frac{1}{\left(1+H_{j}\right)^{3}}-\frac{1}{\left(1+H_{j}\right)^{9}}\right]\left[G\left(\rho_{i}, \tau_{j}\right)-G\left(0, \tau_{j}\right)\right]=0 \text { (19) }
$$


For a given value of $H_{0}$, Eq. (19) can be solved using Newton-Raphson method. Originating from an initial guess $H_{i}^{(0)}$, successive iterates are determined by:

$$
\left\{\begin{array}{c}
(k+1) \\
i
\end{array}\right\}=\left\{H_{i}^{(k)}\right\}-\left[J_{i j}^{(k)}\right]^{-1} R_{i}^{k}(20)
$$

where $\left[J_{i j}{ }^{(k)}\right]$ denotes the Jacobian matrix of sensitivities of the residuals $R_{i}$ with respect to the unknown $H_{j}$. The iteration continues until both max $\left(\left|\left(H_{i}^{(\mathrm{k}+1)}-H_{i}^{(\mathrm{k})}\right) / H_{i}^{(\mathrm{k})}\right|\right)$ and the Euclidean norm of residual $R_{i}$ are less than $10^{-6}$. In order to make an initial estimation of the solution $H_{i}^{(0)}$ that falls within the domain of convergence, the initial $H_{0}$ is selected to be very large (approximately 10), such that the interaction between the sphere and the surface is weak, regardless of the Tabor parameter. In this sense, the deformation of the surface of the half space can be neglected, and the initial guess of separation is

$$
H_{i}=H_{0}+\frac{\rho^{2}}{2 \zeta^{2}}(21)
$$

Once $\zeta$ and $\mu$ are fixed, a given value of $H_{0}$ results a sole solution for $H_{i}\left(H_{0}\right)$, substitution of which will determine both $P\left(H_{0}\right)$ and $\alpha\left(H_{0}\right)$ according to Eq. (17) and (18) respectively. For a new value $\left(H_{0}+\delta H_{0}\right)$, the solution may be obtained by using the existing solution $H_{i}\left(H_{0}\right)$ as the initial guess, i.e. $H_{i}^{(0)}=H_{i}\left(H_{0}\right)$. However, the following initial guess

$$
\left\{H_{i}^{(0)}\right\}=\left\{H_{i}\left(H_{0}\right)\right\}-J^{-1}\left(H_{0}\right) R_{H}\left(H_{0}\right) \delta H_{0}(22)
$$

is often closer for calculating the solution $H_{i}\left(H_{0}+\delta H_{0}\right)$ than using $H_{i}\left(H_{0}\right)$ (Keller, 1977; Feng, 2000), where $R_{H}$ denotes the differentiation of $R$ with respect to $H_{0}$. Therefore, for the cases when the gap is small, with high value of $\zeta$ and $\mu$ where the initial guess $H_{i}{ }^{(0)}=H_{i}\left(H_{0}\right)$ is usually out of range of convergence, solutions can be often obtained by using the initial guess provided by Eq. (22).

The function $G(\rho, \tau)$ exhibits singularity when $\rho=\tau$ and $\theta \rightarrow 0$. In the present study we adopt special treatments to circumvent this issue which is detailed in the Appendix.

\section{Results and discussion}

In the present study, the number for the segments (elements) is $N=500$, and the concurrent number of nodes becomes 501. A further calculation indicates that there is negligibly little difference by setting the upper limit of the integral in Eq. (12) larger than $10\left(R z_{0}\right)^{1 / 2}$. For the simplicity of illustration, our results and discussions herein are restricted to the simplest case where the adhesive contact is between a rigid sphere and an elastic half space with surface tension. This limitation can certainly be cancelled and the results could be generalized to the case of contact between an elastic sphere and a rigid plane or two spheres, as long as the contact region is much smaller than the size of the contacting bodies.

4.1 Load-approach curves for small values of Tabor parameters $\mu$ and $\zeta$ 


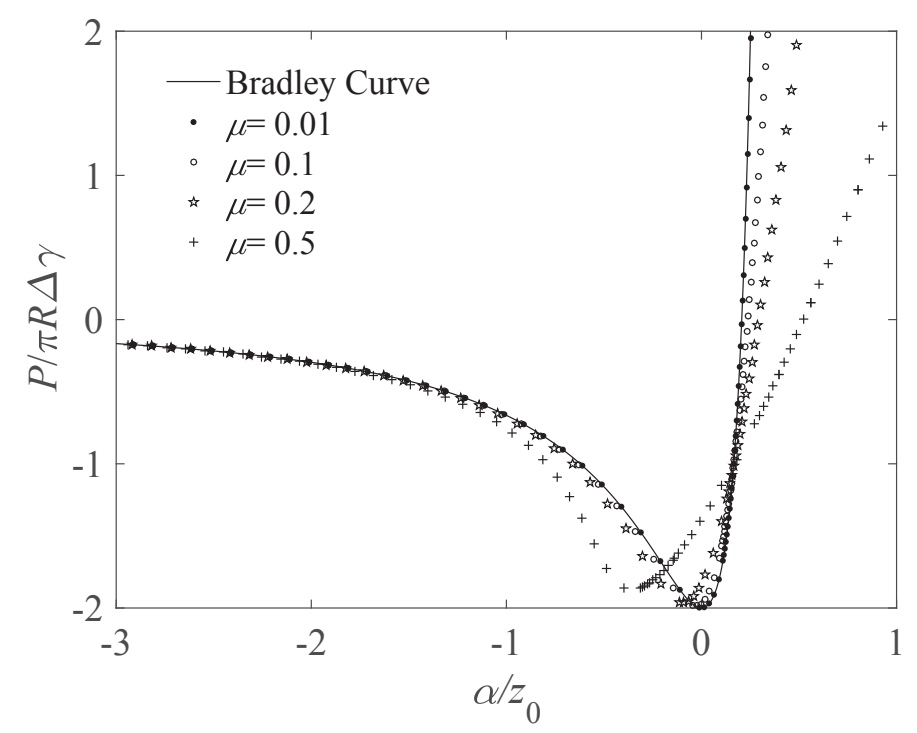

(a)

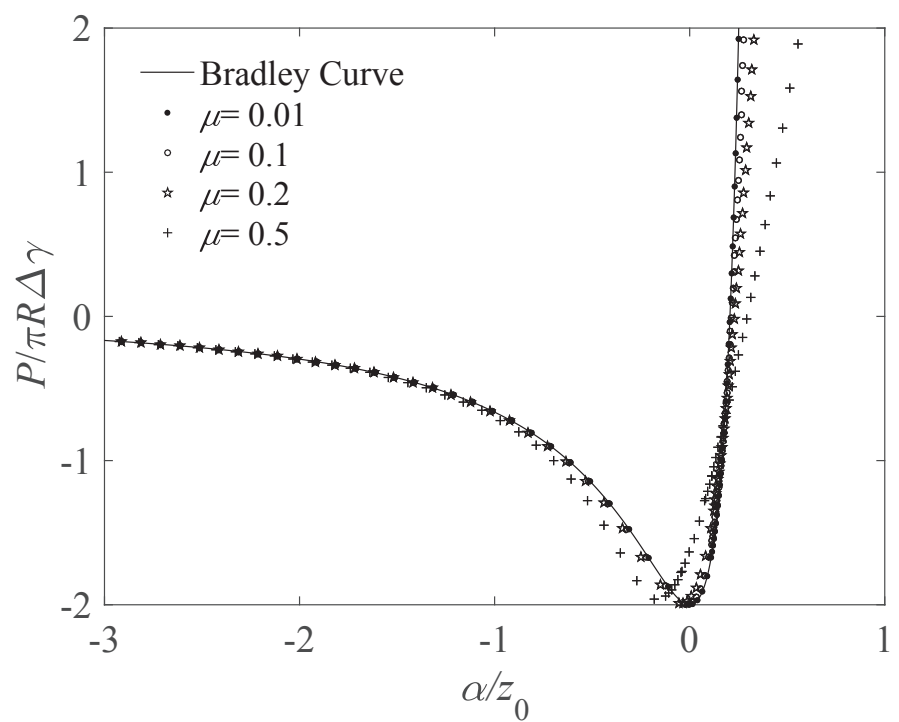

(b) 


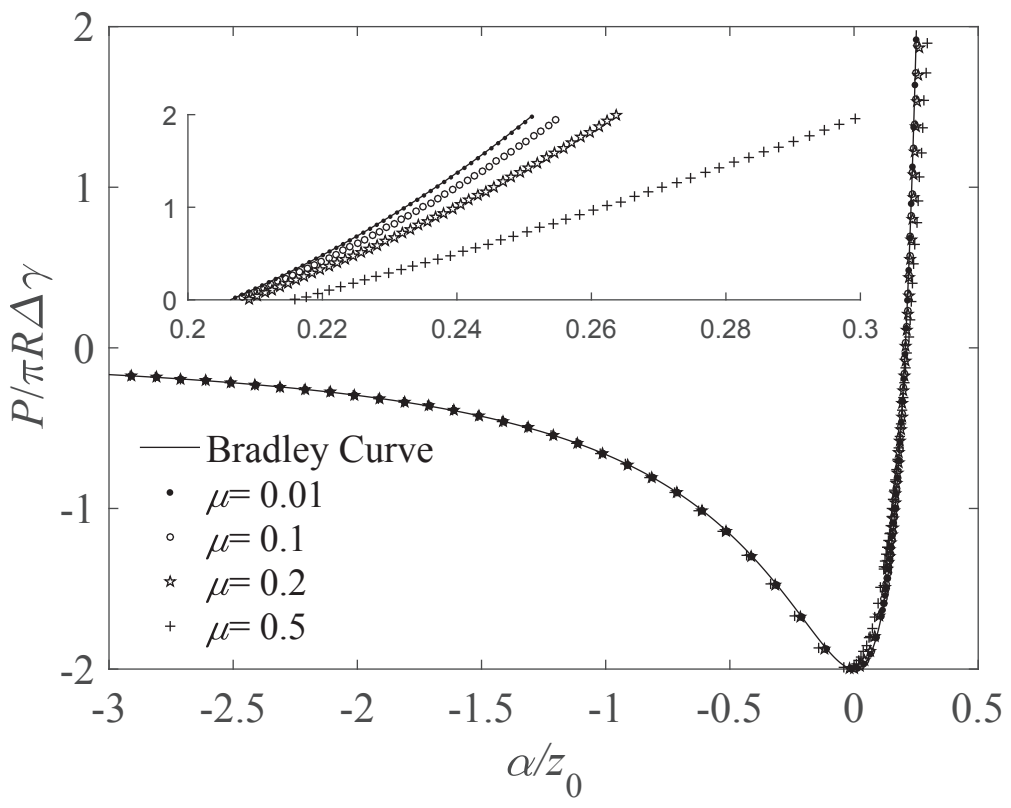

(c)

Fig. 3. Load-approach curves for $(a) \zeta=0.5(b) \zeta=0.1$ and $(c) \zeta=0.01$ corresponding to small value of $\mu$.

Fig. 3 shows the computed load-approach curves in reduced coordinates for different combinations of $\mu$ and $\zeta$. For comparison purposes, we also plot the Bradley curve (Greenwood 1997)

$$
\frac{P}{\pi \Delta \gamma R}=\frac{1-4(1-A)^{6}}{3(1-A)^{8}}(23)
$$

where the half space is rigid. With a fixed $\zeta$, the load-approach curves for small $\mu(<0.2)$ are qualitatively analogous to the curve of L-J potential law for individual molecules (Feng 2000), and the results for $\mu=$ 0.01 (regardless of value of $\zeta$ ) are almost indistinguishable from the Bradley curve, which is the same as the counterpart in the non-surface tension situation (Greenwood 1997; Feng 2000). As shown in Fig. $3(a),(b)$ and (c), with $\zeta$ decreasing, the divergence of the load-approach curves due to the change of $\mu$ becomes less significant, e.g. $\zeta=0.01$, there is inappreciable difference between the four curves. As $\zeta$ is the ratio of $\left(R z_{0}\right)^{1 / 2}$ to $s$, a smaller $\zeta$ indicates greater contribution of the surface tension. In this regard, a strong surface tension would assist the elastic substrate to resist the deformation induced by the adhesive forces, and hence the substrate is likely to behave as a rigid body, irrespective of the elastic modulus of the substrate. Therefore, it is expectable that the load-approach curve with a low $\zeta$ (e.g. 0.01) almost coincides with Bradley curve which describes adhesive contact between two rigid spheres.

\subsection{Analysis on jump-on and jump-off instabilities}

Analogous to the counterpart of the non-surface tension situation, the effect of the short-range component (the second term of Eq. (9)) of intermolecular forces becomes significant when $\mu$ is no longer small, e.g. $\mu \geq$ 1.0 in the present situation. For example, $\mu=\zeta=1.0$, the dashed curve in Fig. 4 exhibits a slight hysteresis phenomenon, as its non-surface tension counterpart (solid line in Fig. 4) presented by Greenwood (1997) and Feng (2000). As pointed out by Feng (2000), the turning points (where $d P / d \alpha \rightarrow \infty$ ), are implied by a variation of the determinant sign of the Jacobian matrix, and indicate switch between stable and unstable equilibrium. The interval of load-approach curve between the turning points should correspond to unstable equilibrium, and the right and left turning points should correspond to the instants of jump-on and jump-off instabilities of contact surfaces, respectively. 


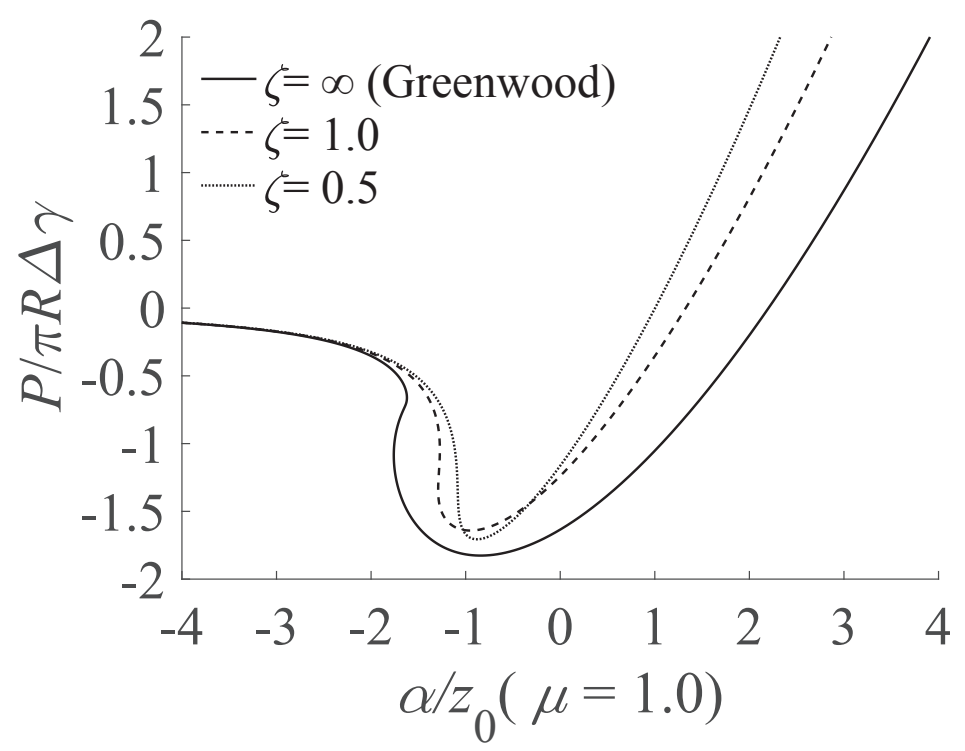

Fig.4. Load-approach curves for $\zeta=0.5,1.0$ and $\infty$, under $\mu=1.0$.

Furthermore, Fig. 4 also indicates that the hysteretic phenomenon is affected by the value of $\zeta$, e.g. it disappears with small $\zeta$ (e.g. $\zeta=0.5$ ) as shown by the dotted curve in Fig. 4. Since a lower $\zeta$ implies that the relative contribution of surface tension is greater, it is understandable that stronger surface tension enables the half space to behave like a stiffer material, tending to vanish the occurrence of the hysteresis. In this regard, this also explains why the instant of jump-on or jump-off is postponed (i.e. the absolute value of the corresponding approach $\alpha$ decreases) as $\zeta$ decreases, by comparing the solid and dashed curves in Fig. 4. This can be quantitatively interpreted as follows. With a small value of $\mu$ (e.g. $\mu \leq 1.0$ ), it is reasonable to assume that the deformation is negligibly affected by the $\mathrm{L}-\mathrm{J}$ surface interaction (Song and Komvopoulos 2011), and thus one has

$$
h(t)=h_{0}+\frac{t^{2}}{2 R}(24)
$$

According to Eq. (11), the central surface displacement $w(0,0)$ of the half space is given by:

$$
w(0,0)=\int_{t=0}^{\infty} \frac{\pi p(t) t}{2 \sigma} \psi(\stackrel{t}{s}) d t(25)
$$

Combining Eq. (9), (24) and (25) yields:

$$
w(0,0)=\frac{4 \pi \Delta \gamma}{3 \varepsilon \sigma} \int_{t=0}^{\infty} t\left[\left(\frac{z_{0}}{h_{0}+t^{2} / 2 R}\right)^{3}-\left(\frac{z_{0}}{h_{0}+t^{2} / 2 R}\right)^{9}\right] \psi\left(\frac{t}{s}\right) d t(26)
$$

Noting the geometric relationship, one has

$$
-\alpha=h_{0}+w(0,0)(27)
$$

As indicated by Song and Komvopoulos (2011), at the instant of jump-on (or jump-off), an infinitesimal relative approach $d \alpha$ could induce upward (downward) displacement of the surface to jump into (off) the sphere. In this sense, requirement for the jump-on (jump-off) condition can be mathematically expressed as

$$
\frac{d \alpha}{d h_{0}} \rightarrow 0(28)
$$

Considering Eq. (27), one has 


$$
\frac{d w(0,0)}{d h_{0}} \rightarrow-1
$$

If one uses dimensionless parameters $u=t\left(\left(R z_{0}\right)^{1 / 2}\right.$ and $h_{0}{ }^{*}=h_{0} / z_{0}$ (i.e. $h_{0}{ }^{*}=1+H$ ) instead, differentiating Eq. (26) with respect to $h_{0}$ leads to

$$
\frac{8 \pi \zeta \mu^{3 / 2}}{3} \int_{u=0}^{\infty} u\left[9\left(h_{0}^{*}+\frac{u^{2}}{2}\right)^{-10}-3\left(h_{0}^{*}+\frac{u^{2}}{2}\right) \quad\right] \psi(\zeta u) d u=-1(30)
$$

Again, we set the upper limit of the integral equation in Eq. (30) to be 10 as detailed previously, and plot the dependence of the critical central gap $h_{0}{ }^{*}$ on the Tabor parameter corresponding to four different values of $\zeta$, as shown in Fig. 5.

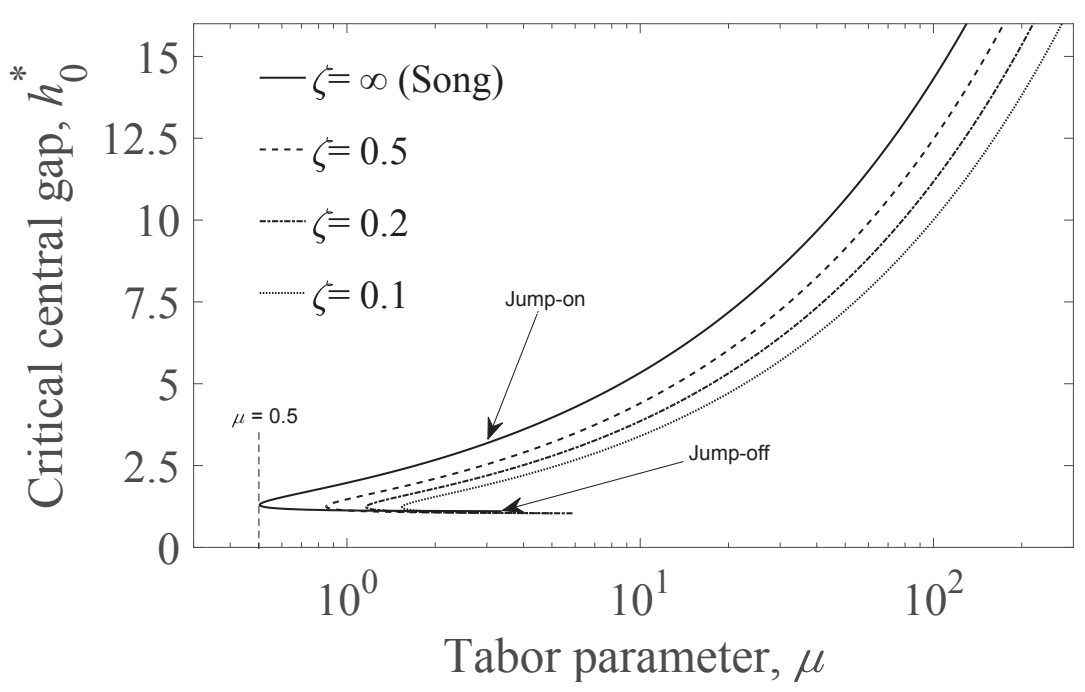

Fig. 5. Critical central gap at the instant of jump instabilities versus Tabor parameter corresponding to four different values of $\zeta$.

Fig. 5 presents an intuitive understanding on the effect of the parameter $\zeta$ on the instability behavior. For a fixed value of $\zeta$, the leftmost point of each curve denotes the threshold value of Tabor parameter where hysteresis appears, and below this threshold no instability would be encountered. Each curve is divided by this leftmost point into two parts, with upper and lower bifurcations representing jump-on and jump-off instabilities respectively. It can be seen that the existence of surface tension increases the threshold of the Tabor parameter to induce hysteresis, with lower values of $\zeta$ corresponding to higher values of critical $\mu$. Furthermore, for a fixed $\mu$, a lower central gap $h_{0}{ }^{*}$ is observed to initiate jump instability of curve with lower $\zeta$, which is indeed consistent with previous analysis. Although Eq. (30) is based on the assumption of small Tabor parameter, further calculation by setting $\mu$ to a be high value (e.g. $\mu=5.0)$ indicates that the effect of parameter $\zeta$ on the position of jump instability still holds, as illustrated in Fig. 6. 


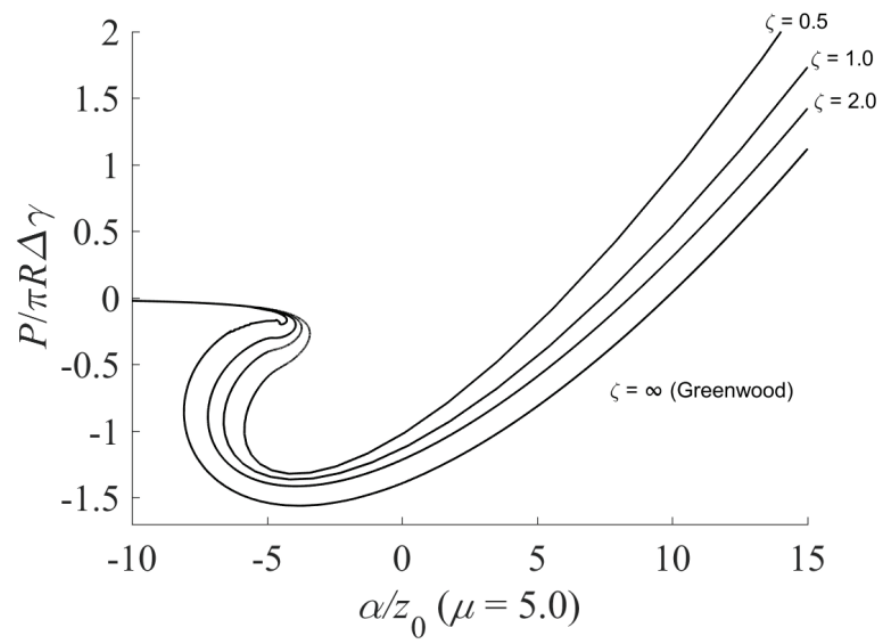

Fig. 6. Load-approach curves in reduced coordinates corresponding to four values of $\zeta$ at high values of Tabor parameter (e.g. $\mu=5.0)$.

\subsection{Pull-off force and transition between JKR model and Young-Dupre law}

Fig. 6 appears to show that the pull-off force (the minimum value of the dimensionless force in the load-approach curve) decreases monotonically as $\zeta$ decreases, however, this is not the whole story. Fig. $7(a)$ shows that at $\mu=5.0$, there is a non-monotonic trend of the pull-off force versus the parameter $\zeta$. The absolute value of the pull-off force decreases first and then increases as $\zeta$ increases from 0.001 to 2.0. It is worth noting that at a very low $\zeta$ (e.g. 0.001), the load-approach curve almost coincides with the Bradley curve regardless of high value of Tabor parameter. In other words, when half space is subject to high surface tension $\sigma$ (low value of $\zeta$ ), the adhesive contact behavior actually imitates that between two rigid spheres despite very strong adhesive forces (i.e. high value of $\mu$ ). On the other hand, with a large $\zeta($ e.g. $\zeta=2.0)$, the load-approach curve approaches that of JKR theory as shown in Fig. 7 $(c)$ and $(d)$, as expected.

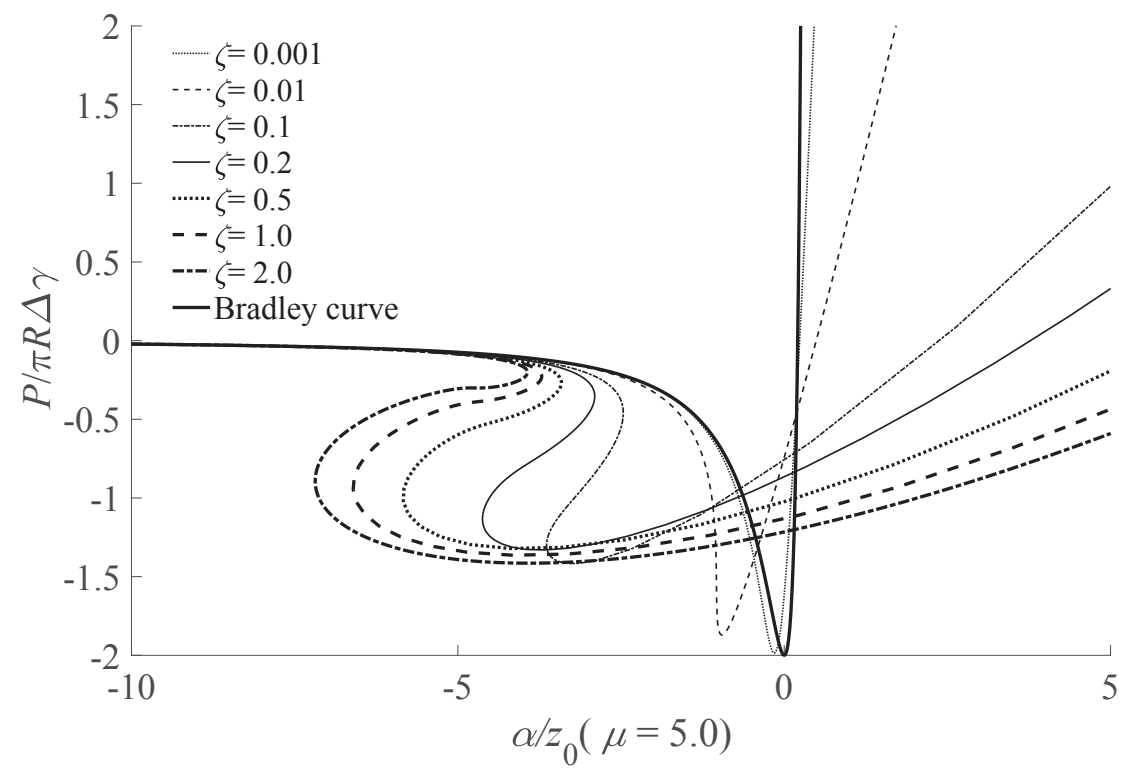

(a) 


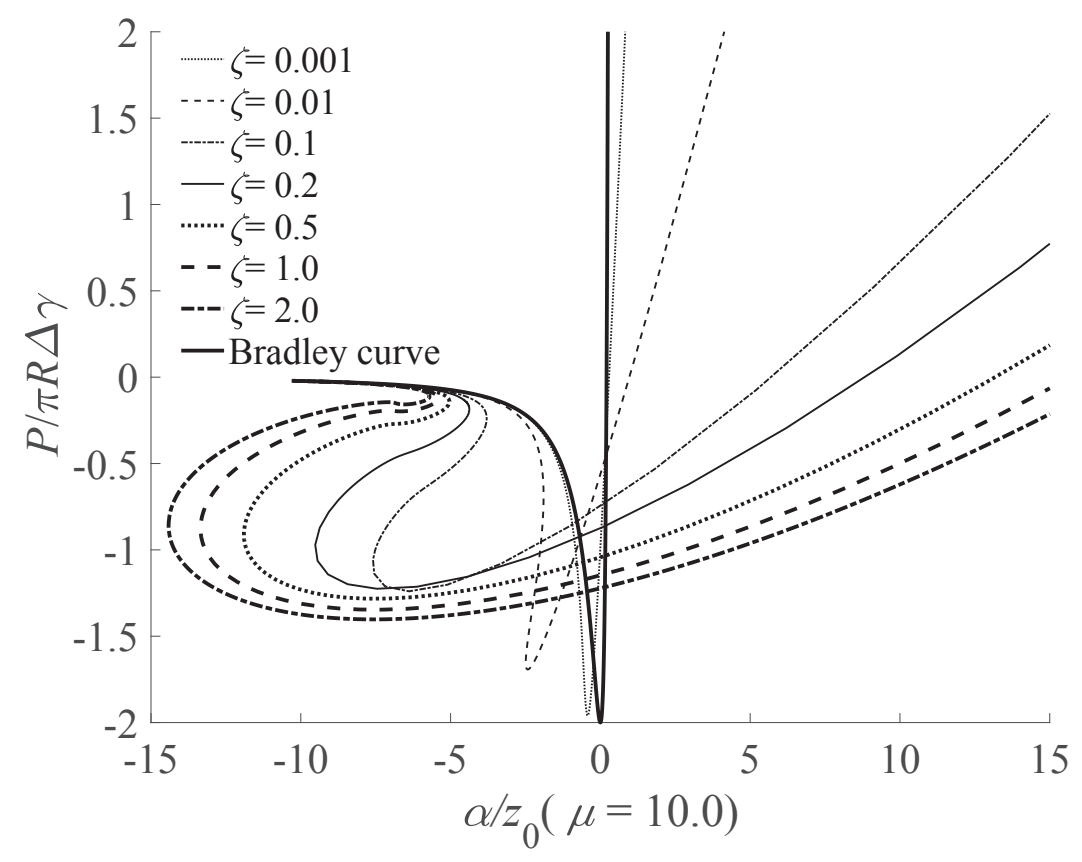

(b)

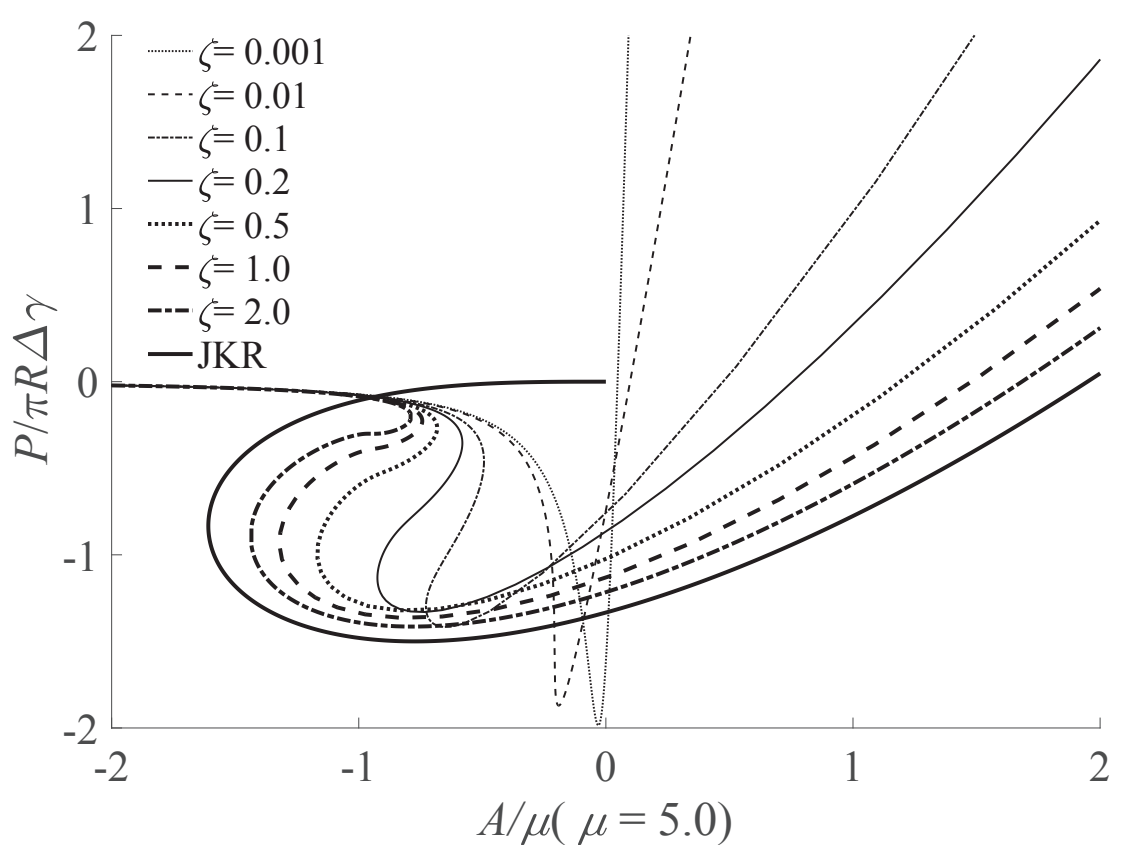

(c) 


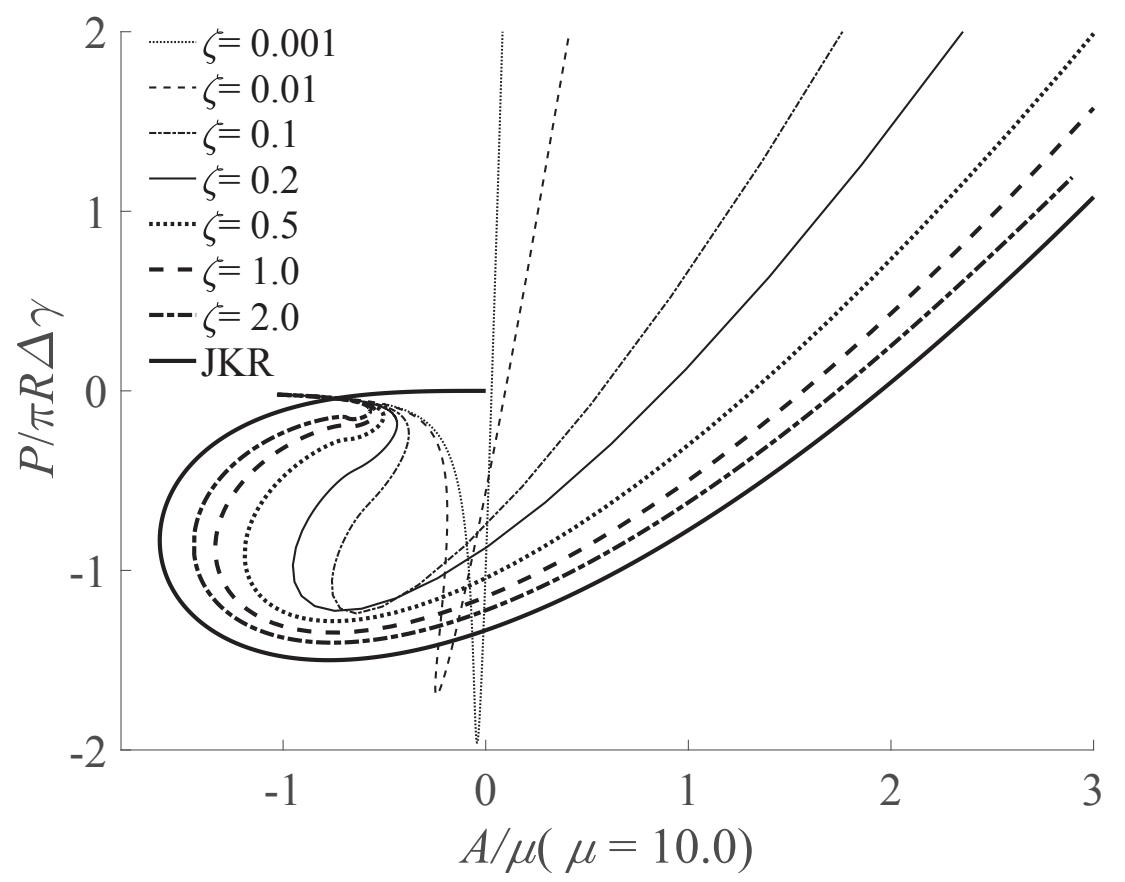

(d)

Fig. 7. Load-approach curves for $(a) \mu=5.0$ and $(b) \mu=10.0$. (c) and $(d)$ illustrate the variation of load with approach, together with JKR theory.

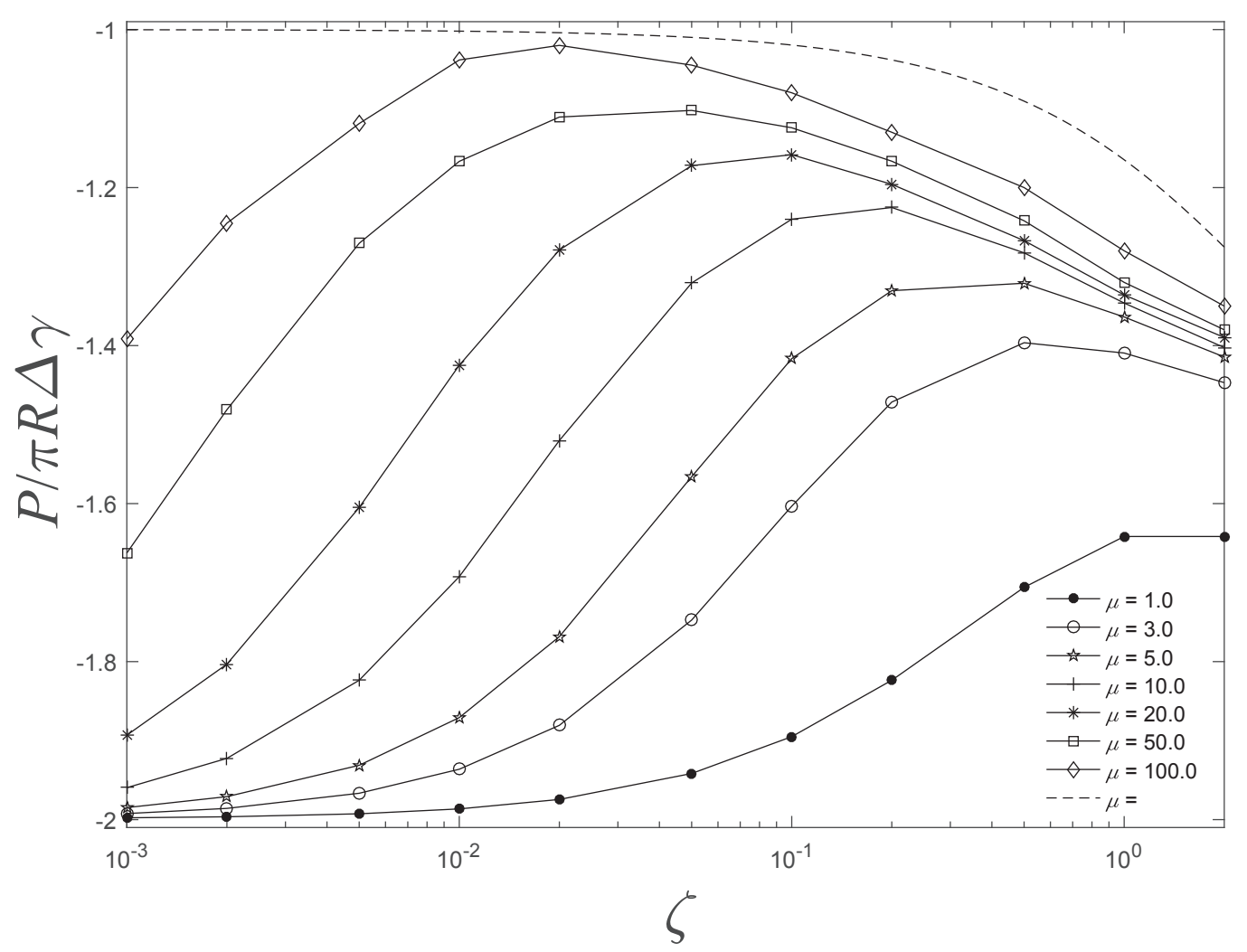

Fig. 8. Pull-off force as a function of the parameter $\zeta$ under different fixed Tabor parameters. 
Fig. 8 presents an intuitional illustration of the effect of $\zeta$ on the pull-off forces with different $\mu$. In a nonsurface situation, for high value of Tabor parameters (e.g. $\mu=3,5,10$ and 20), both Greenwood (1997) and Feng (2000) showed the pull-off forces are marginally lower than -1.5 . However, for the same high values of Tabor parameters, the pull-off forces in the case of surface tension are higher than -1.5 , and the increasing of the pull-off force is ascribed to the effect of surface tension.

The non-monotonic dependence of pull-off force on $\zeta$ is very conspicuous for a high $\mu$ (e.g. $\mu=3,5,10$ and 20), and there is a peak point on pull-off force versus $\zeta$ curve. After passing this point from right to left, the pull-off force dramatically drops to -2 . As the Tabor parameter increases, this peak point moves in upper left direction as shown in Fig. 8. As $\mu$ further increases, it can be seen that the peak point keeps shifting in upper left direction and asymototically approaches the point $(0,-1)$. In this regard, it is rational to suppose that this peak point will overlap with point $(0,-1)$ if the Tabor parameter tends towards infinity, as illustrated by the dashed curve in Fig. 8. In other words, with infinite $\mu$ (the corresponding intermolecular forces could be regarded as a delta function), the pull-off force decreases monotonically from -1 to -1.5 as $\zeta$ increases from zero to infinity, as illustrated by the dashed line in Fig. 8. In fact, this rational hypothesis is consistent with the former study by Hui et al. (2015). They considered intermolecular forces as short-range (in fact, they are treated as a delta function), and indicated that $P / \pi \Delta \gamma R=-1.5$ and -1 correspond to JKR theory and Young-Dupre law, respectively. Hui et al. (2015) used the parameter $\omega=\sigma(G R)^{-2 / 3}(9 \pi \Delta \gamma / 4)^{-1 / 3}$ to characterize the transition between these two limits, with small and large $\omega$ corresponding to JKR theory and Young-Dupre law, respectively. According to the definition of $\mu$ and $\zeta$, it is not difficult for one to obtain

$$
\omega \propto \frac{\mu^{-1 / 2}}{\zeta}(31)
$$

In the present study, as $\mu \rightarrow \infty$ and $\zeta \rightarrow 0$ where both adhesion and surface tension dominate the elasticity of the half space, one has $\omega \rightarrow \infty$ because $\zeta$ is infinitesimal of higher order compared with $\mu^{-1 / 2}$ according to Eq. (31). In this sense, the deformation attributed to adhesion is primarily resisted by the surface tension, and thus the contact behavior exhibits "liquid" limit, i.e. described by the Young-Dupre law (Style et al., 2013; Hui et al., 2014). However, different results are presented in present study compared with the counterpart in the study by Hui el al., (2015). For a combination of high $\mu$ and low $\zeta$, as long as they are finite and nonzero, the load-approach curve almost overlaps with Bradley curve as illustrated in Fig. $7(a)$ and $(b)$, and the pulloff tends towards -2 as shown in Fig. 8. This can be interpreted as follows. Since both $\mu$ and $\zeta$ are finite and nonzero, the elasticity of the half space cannot be neglected, and it should be distinguishable with "liquid" limit where the elastic modulus is null. On the other hand, a finite Tabor parameter implies the intermolecular forces cannot be regarded as a delta function. In this situation, the former context indicates that the half space behaves as a rigid body despite a large value of $\mu$, which implies that surface tension also dominates the adhesion. Therefore, in a solid with finite elastic modulus, this domination results in another pull-off force limit equal -2 . In summary, with finite elasticity and high $\mu$, as $\zeta$ decreases, the pull-off force increases from the JKR limit, approaches the Young-Dupre limit, and decreases dramatically to the Bradley limit; when the elastic modulus of solid tends towards zero ("liquid" limit), the pull-off force changes from JKR limit to Young-Dupre limit monotonically as the parameter $\omega$ increases.

\subsection{Particle surface profiles and pressure distributions}

In the contact between a rigid sphere and an elastic half space, it is of great importance to investigate the pressure distribution and the associated deformation of the half space. For the purpose of convenience, we define a dimensionless local pressure $p^{*}(\rho)$ as

$$
p^{*}(\rho)=-\frac{8}{3}\left\{[1+H(\rho)]^{-3}-[1+H(\rho)]^{-9}\right\}(32)
$$


which could be viewed as the local pressure $p(r)$ in Eq. (9) scaled by $\Delta \gamma / z_{0}$. In the following figures, since $H$ and $r$ are scaled differently, the surface profile of the half space plotted as $H$ versus $r /\left(R z_{0}\right)^{1 / 2}$ might exaggerate the actual deformation appreciably.

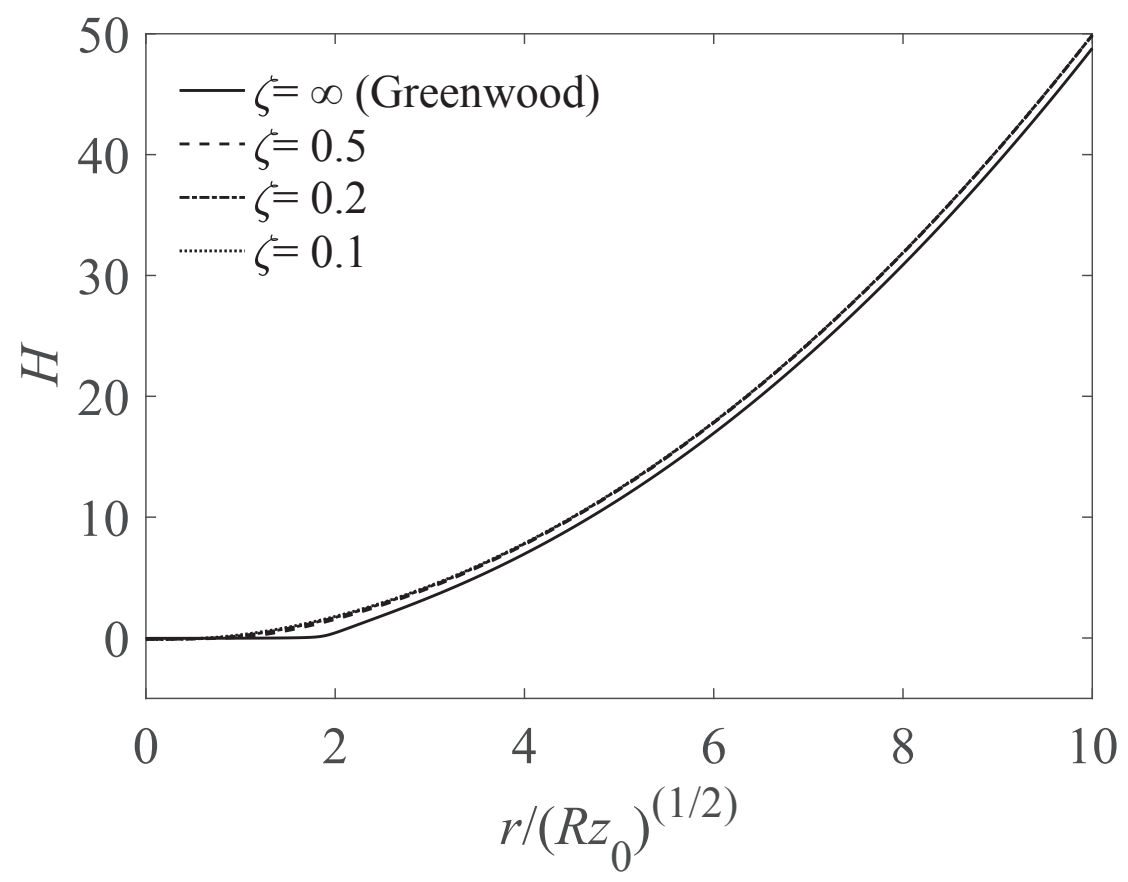

(a)

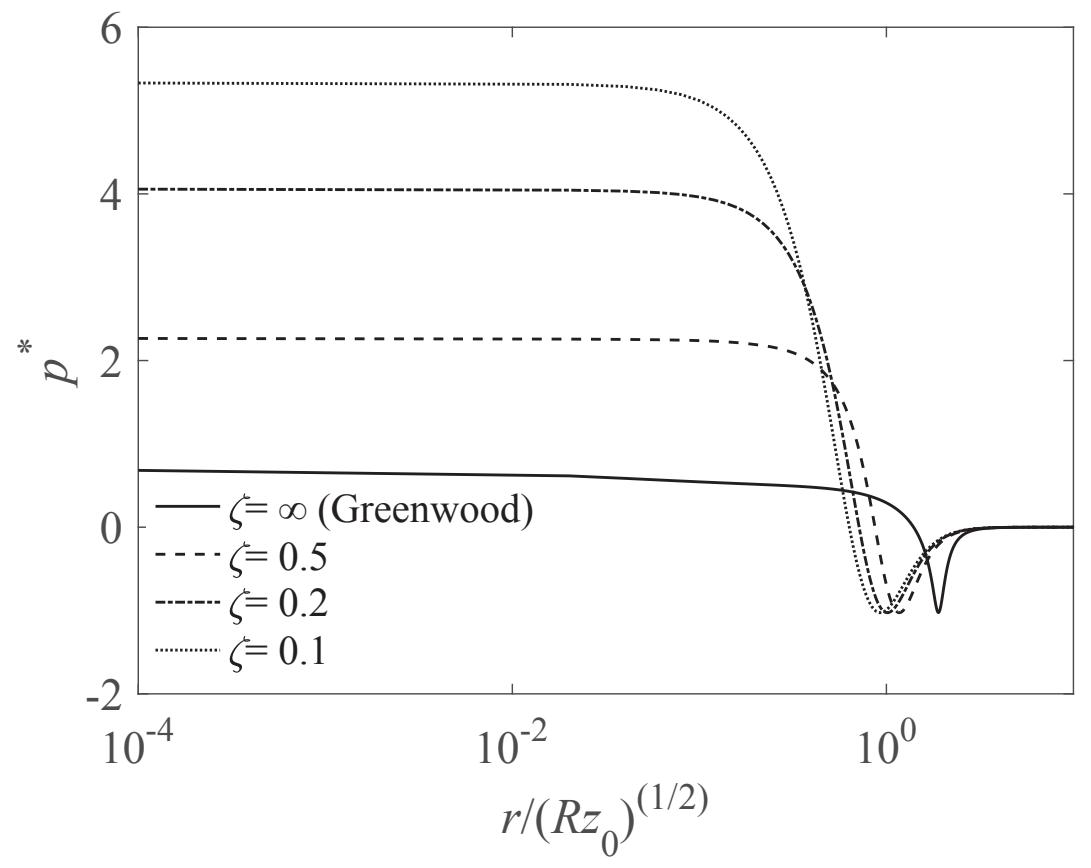

(b)

Fig. 9. Deformation profiles (a) and pressure distributions (b) for $\mu=1.0$ at $\alpha=0$. 


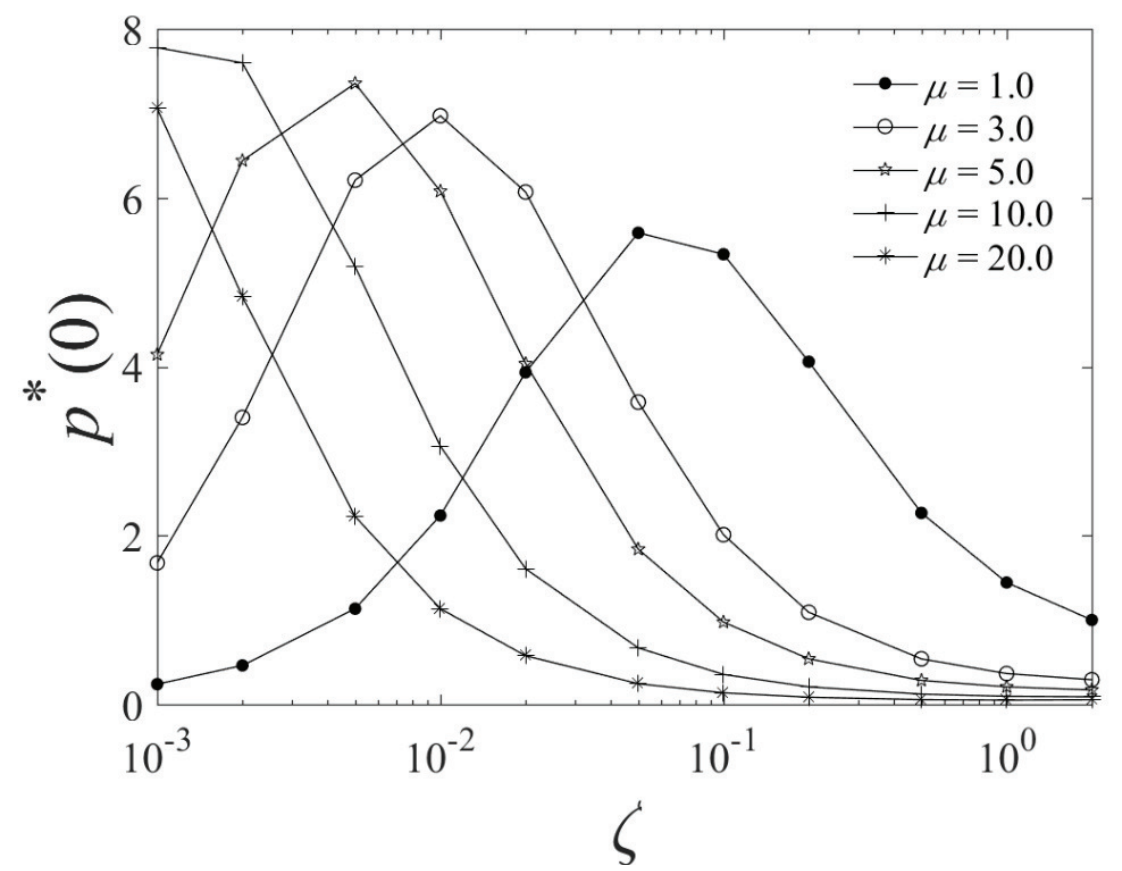

Fig. 10. The dependence of central pressure on the parameter $\zeta$ under different fixed Tabor parameters corresponding to zero approach.

The case of $\alpha=0$ is of particular interest, and it indicates the apex of the rigid sphere reaches the zero force position $z=-z_{0}$, which is equivalent to the situation when two rigid spheres "contact" at a mathematical point (Feng, 2000). Fig. $9(a)$ and $(b)$ show the surface profiles and pressure distributions for a small value of $\mu$ (e.g. $\mu=1)$ at $\alpha=0$, respectively. For cases of surface tension $(\zeta=0.1,0.2$ and 0.5$)$, the deformation of the half space is less significant than that of the non-surface tension case, and indistinguishable difference is observed between the deformation profiles corresponding to different values of $\zeta$. This is in accordance with our expectation since the surface tension would help the half space to resist the deformation caused by the adhesive forces. However, the pressure distributions corresponding to different $\zeta$ are discernible, which are also distinguishable from the non-surface tension case. In the case of $\mu=1$, for the four discrete values of $\zeta$ $=0.1,0.2,0.5$ and $\infty$, a smaller $\zeta$ would result in a higher central pressure, i.e. $p^{*}(0)$, and a smaller contact radius, which is defined as the location where the pressure reaches its minimum (Greenwood, 1997). Such a significant alternation in central pressure and contact radius in response to a slight change in the deformation profiles indicates that the repulsive component of the L-J potential law is highly sensitive to the surface tension. The minimum pressure seems to be a constant very close to $p^{*}=-1$, which is the same as its counterpart in non-surface tension (Feng 2000). A further investigation on the central pressure in relation to more values of $\zeta$ indicates that, under a small $\mu$, e.g. $\mu=1$, the monotonic dependence of $p^{*}(0)$ on $\zeta$ never holds for smaller $\zeta$ (e.g. $\zeta=0.01$ and 0.001 ), as shown in Fig. 10. Nevertheless, large or small values of Tabor parameter $\mu$ could expand or curtail this monotonic interval, respectively. 


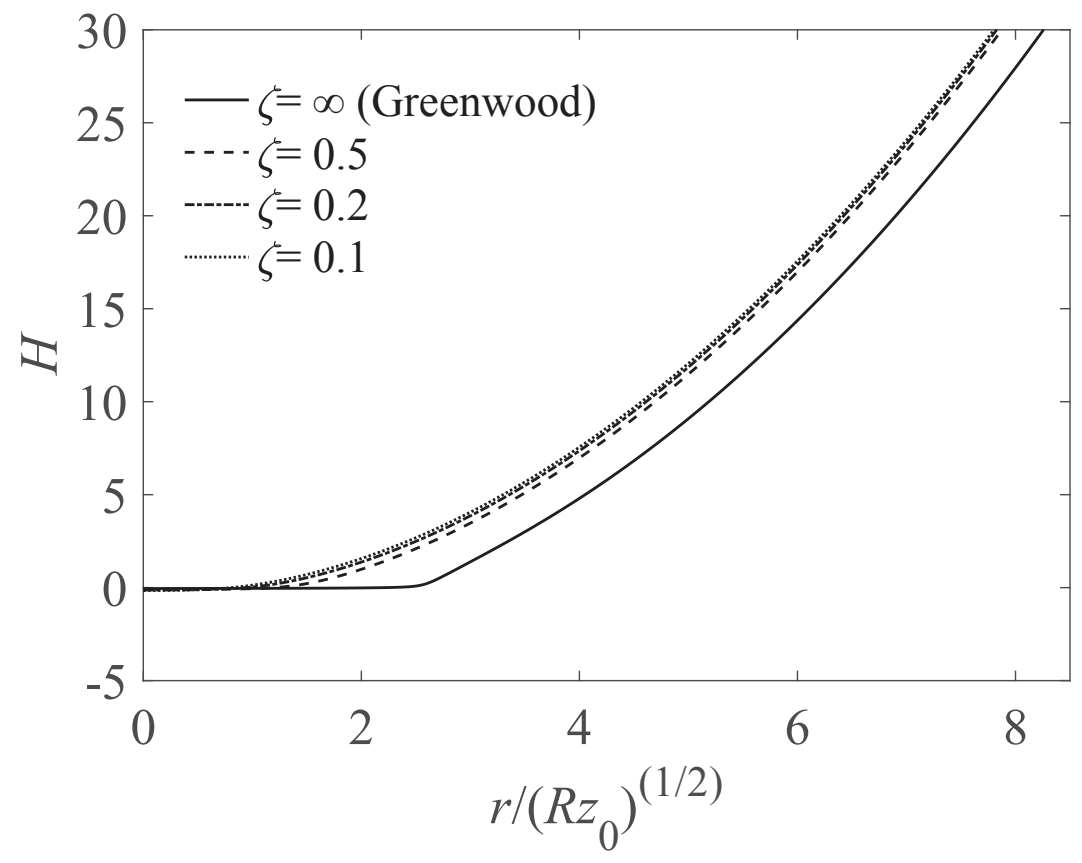

(a)

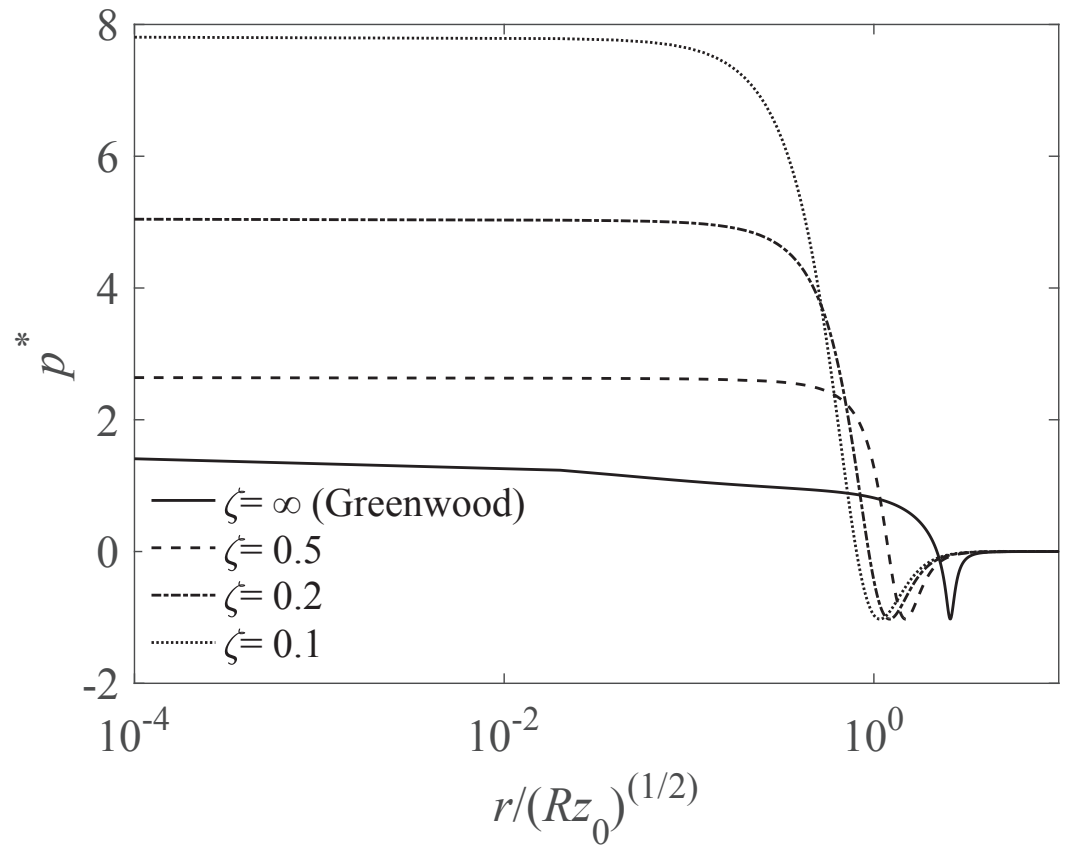

(b)

Fig. 11. Deformation profiles $(a)$ and pressure distributions $(b)$ for $\mu=1.0$ at $P=0$. 


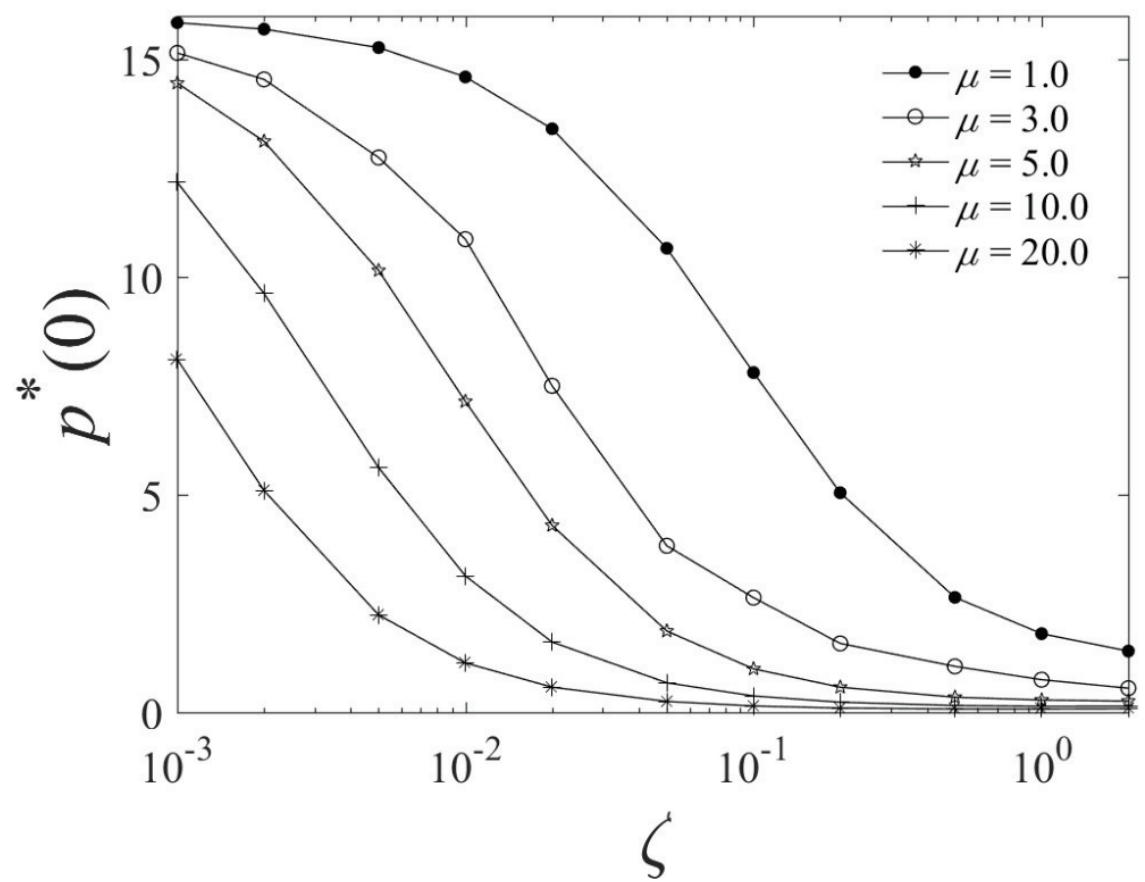

Fig. 12. The dependence of central pressure on the parameter $\zeta$ under different fixed Tabor parameters corresponding to zero force.

Another special case of interest is when $P=0$, which indicates that the repulsive and attractive force components of the intermolecular interactions are in mechanical equilibrium in the absence of any externally applied load. Fig. $11(a)$ and $(b)$ illustrate the deformation profile and pressure distribution respectively in relation to $\mu=1.0$ in this situation. It can be seen that the features of deformation profile and pressure distribution under zero applied force are analogous to that of zero approach. However, at $P=0$, the central pressure $p^{*}(0)$ is monotonically decreasing with increasing $\zeta$. In addition, the minimum pressure remains at a constant value of -1 , regardless the variation of $\zeta$.

\section{Conclusions}

In the present study, we investigated the effect of surface tension on the adhesive contact between a rigid sphere and an elastic half space, where the interfacial adhesion was represented by the Lennard-Jones potential law. This adhesive contact problem was transformed into a nonlinear integral equation which was solved by Newton-Raphson method. Compared with previous studies where the interfacial adhesion is attributed to short-range intermolecular forces (exactly speaking, they are treated as a delta function), the present study ascribes the interfacial adhesion to long-range forces described by L-J potential law. As the result, more details of the adhesive contact behavior are explored. The existence of surface tension indeed assists the half space to resist adhesion-induced deformation. With a strong effect of surface tension, the adhesive contact behavior between the rigid sphere and the half space with finite elastic modulus even resembles that between two rigid spheres, irrespective of the adhesion strength. Surface tension alters the features of jump-on and jump-off instabilities. Compared with the non-surface tension situation, a higher Tabor parameter is needed to initiate jump instability. For a fixed Tabor parameter, the jump-on instability is postponed when the sphere approaches the half space, while the jump-off instability is brought forward when the sphere retracts. The pull-off force is also affected by the surface tension. For a half space with finite elastic modulus subject to long-range intermolecular forces, the pull-off force increases to the peak value and then decreases dramatically to -2 as the parameter $\zeta$ reduces. However, a rational assumption indicates that when the elastic modulus of the half space approaches zero, the pull-off force increases monotonically to -1 with $\zeta$ decreasing, which is consistent with a previous study by Hui et al. (2015). 


\section{Acknowledgements}

The authors would like to acknowledge the funding support to Dr Xinyao Zhu as a postdoctoral research fellow from the EU Horizon 2020 (645239) project.

\section{Appendix}

If $\rho=\tau$, Eq. (13) could be rewritten as

$$
G(\rho, \rho)=\int_{\theta=0}^{\pi} \psi\left[2 \rho \sin \left(\frac{\theta}{2}\right)\right] d \theta(\text { A.1) }
$$

If $\theta \rightarrow 0^{+}$, one has

$$
\psi\left[2 \rho \sin \left(\frac{\theta}{2}\right)\right] \approx-Y_{0}\left[2 \rho \sin \left(\frac{\theta}{2}\right)\right] \approx-\frac{2}{\pi}\left\{\ln \left[\rho \sin \left(\frac{\theta}{2}\right)\right]+\gamma\right\} \text { (A.2) }
$$

where $\gamma$ denotes the Euler-Mascheroni constant, i.e. $\gamma \approx 0.5772$. The singular one-dimensional integral Eq. (A.1) could be changed into non-singular two-dimensional integrals by virtue of the identities (Feng 2000):

$$
\int_{x=0}^{1} f(x) \ln (x) d x=-\int_{x=0}^{1} \int_{y=0}^{1} f(x y) d x d y(\text { A.3) }
$$

where $f(x)$ is a well-behaving function of $x$ on $0 \leq x \leq 1$. 


\section{References}

Bradley, R.S., 1932. The cohesive force between solid surfaces and the surface energy of solids. Phil. Mag. $13,853-862$.

Bian, J., Wang, G., Feng, X., 2012. Atomistic calculations of surface energy of spherical copper surfaces. Acta Mech. Solida Sin. 25 557-561.

Cammarata, R.C., 1994. Surface and interface stress effects in thin films. Prog. Surf. Sci. 46, 1-38.

Cao, Z., Stevens, M.J., Dobrynin, A.V., 2014. Adhesion and wetting of nanoparticles on soft surfaces.

Macromolecules 47, 3203-3209.

Chakrabarti, A., Chaudhury, M.K., 2013. Direct measurement of the surface tension of a soft elastic hydrogel: exploration of elastocapillary instability in adhesion. Langmuir 29, 6926-6935.

Chen, S.H., Yao, Y., 2014. Elastic Theory of Nanomaterials Based on Surface-Energy Density. J. Appl. Mech. 81,121002.

Chen, T.Y., Chiu, M.S., Weng, C.N., 2006. Derivation of the generalized Young-Laplace equation of curved interfaces in nanoscaled solids. J. Appl. Phys. 100, 074308.

Feng, J.Q., 2000. Contact behavior of spherical elastic particles: a computational study of particle adhesion and deformations. Colloids Surf. A, Physicochem. Eng. Asp. 172, 175-198.

Greenwood, J.A., 1997. Adhesion of elastic spheres. Proc. R. Soc. Lond. A 453, 1277-1297.

Gurtin, M.E., Murdoch, A.I., 1975. A continuum theory of elastic material surfaces. Arch. Ration. Mech. Anal. 57, 291-323.

Gurtin, M.E., Weissmuller, J., Larche, F., 1998. A general theory of curved deformable interfaces in solids at equilibrium. Philos. Mag. A 78, 1093-1109.

Hajji, M.A., 1978. Indentation of a membrane on an elastic half space. J. Appl. Mech. 45, 320-324. 
Hui, C.Y., Liu, T.S., Salez, T., Raphael, E., Jagota, A., 2015. Indentation of a rigid sphere into an elastic substrate with surface tension and adhesion. Proc. R. Soc. A 471, 20140727.

Israelachvili, J.N., 1992. Intermolecular and Surface Forces, second ed., Academic Press, San Diego, CA.

Johnson, K.L., Kendall, K., Roberts, A.D., 1971. Surface energy and the contact of elastic solids. Proc. R. Soc. Lond. A 324, 301-313.

Keller, H.B., 1977. Numerical solution of bifurcation and nonlinear eigenvalue problems, in: P. Rabinowitz (Ed.), Applications of Bifurcation Theory, Academic Press, New York.

Long, J.M., Wang, G.F., Feng, X.Q., Yu, S.W., 2016. Effects of surface tension on the adhesive contact between a hard sphere and a soft substrate. Int. J. Solids Struct. 84, 133-138.

Mogilevskaya, S.G., Pyatigorets, A.V., Crouch, S.L., 2011. Green function for the problem of a plane containing a circular hole with surface effects. J. Appl. Mech. 78, 021008.

Rimai, D., Quesnel, D., Busnaina, A., 2000. The adhesion of dry particles in the nanometer to micrometersize range. Colloids Surf. A, Physicochem. Eng. Asp. 165, 3-10.

Salez, T., Benzaquen, M., Raphaël, É., 2013. From adhesion to wetting of a soft particle. Soft Matter. 9, 10699-10704.

Shenoy, V.B., 2005. Atomistic calculations of elastic properties of metallic fcc crystal surfaces. Phys. Rev. B 71, 094104.

Song, Z., Komvopoulos, K., 2011. Adhesion-induced instabilities in elastic and elastic-plastic contacts during single and repetitive normal loading. J. Mech. Phys. Solids 59, 884-897.

Style, R.W., Hyland, C., Boltyanskiy, R., Wettlaufer, J.S., Dufresne, E.R., 2013. Surface tension and contact with soft elastic solids. Nat. Commun. 4, 2728-2733.

Xu, X.J., Jagota, A., Hui, C.Y., 2014. Effects of surface tension on the adhesive contact of a rigid sphere to a compliant substrate. Soft Matter 10, 4625-4632.

Yang, F.Q., 2004. Size-dependent effective modulus of elastic composite materials: spherical nanocavities at dilute concentrations. J. Appl. Phys. 95, 3516-3520. 